\title{
All-organic fluorine-free superhydrophobic bulk material with mechanochemical robustness and photocatalytic functionality
}

\author{
Liu, Shanqiu ; Wan, Wenchao ; Zhang, Xiaotian ; De Crema, Alessandro ; Seeger, Stefan
}

\begin{abstract}
Artificial superhydrophobic surface has attracted extensive research interests and versatile applications have been demonstrated. However, such surfaces feature mechanical and chemical susceptibility and lose superhydrophobicity after oily contamination. So far, developing a simple method to fabricate superhydrophobic material featuring mechanochemical robustness along with oil fouling resistance remains challenging. Here we describe a straightforward approach to fabricate superhydrophobic bulk composite materials with photocatalytic functionality that simultaneously feature mechanochemical robustness and oil fouling resistance. The obtained bulk material shows mechanical robust superhydrophobicity throughout its whole volume and maintains excellent water repellency after exposure to highly corrosive liquids and organic solvents. Notably, the bulk material possesses photocatalytic selfcleaning functionality, which enables the elimination of the oily contaminations on the substrate surface, leading to the regeneration of its superhydrophobicity. The robust superhydrophobicity and functionality provide the developed bulk material potential applications in harsh chemical and oily environments where robustness is required.
\end{abstract}

DOI: https://doi.org/10.1016/j.cej.2019.123969

Posted at the Zurich Open Repository and Archive, University of Zurich ZORA URL: https://doi.org/10.5167/uzh-183312

Journal Article

Accepted Version

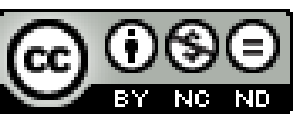

The following work is licensed under a Creative Commons: Attribution-NonCommercial-NoDerivatives 4.0 International (CC BY-NC-ND 4.0) License.

Originally published at:

Liu, Shanqiu; Wan, Wenchao; Zhang, Xiaotian; De Crema, Alessandro; Seeger, Stefan (2020). All-organic fluorine-free superhydrophobic bulk material with mechanochemical robustness and photocatalytic functionality. Chemical Engineering Journal, 385:123969.

DOI: https://doi.org/10.1016/j.cej.2019.123969 


\title{
All-organic Fluorine-free Superhydrophobic Bulk Material with Mechanochemical Robustness and Photocatalytic Functionality
}

\author{
Shanqiu Liu, Wenchao Wan, Xiaotian Zhang, Alessandro De Crema and Stefan Seeger* \\ Department of Chemistry, University of Zurich, Winterthurerstrasse 190, CH-8057 Zurich, \\ Switzerland. \\ *Corresponding author: sseeger@ chem.uzh.ch
}

\begin{abstract}
Artificial superhydrophobic surface has attracted extensive research interests and versatile applications have been demonstrated. However, such surfaces feature mechanical and chemical susceptibility and lose superhydrophobicity after oily contamination. So far, developing a simple method to fabricate superhydrophobic material featuring mechanochemical robustness along with oil fouling resistance remains challenging. Here we describe a straightforward approach to fabricate superhydrophobic bulk composite materials with photocatalytic functionality that simultaneously feature mechanochemical robustness and oil fouling resistance. The obtained bulk material shows mechanical robust superhydrophobicity throughout its whole volume and maintains excellent water repellency after exposure to highly corrosive liquids and organic solvents. Notably, the bulk material possesses photocatalytic selfcleaning functionality, which enables the elimination of the oily contaminations on the substrate surface, leading to the regeneration of its superhydrophobicity. The robust superhydrophobicity and functionality provide the developed bulk material potential applications in harsh chemical and oily environments where robustness is required.
\end{abstract}

Keywords: superhydrophobic bulk material, mechanochemical robustness, photocatalytic functionality, self-cleaning, multifunctional 


\section{Introduction}

Superhydrophobicity of solid substrate surfaces, which is inspired by nature such as, lotus leaves [1], water strider legs [2], dragonfly wings [3] and so on, has attracted tremendous interests in both academia and industry due to its remarkable applications in self-cleaning [4-9], anti-icing [10, 11], anti-corrosion, [12] water-oil separation [13-18] and energy-saving fluid transport, $[19,20]$ etc. To date, various artificial surfaces featuring excellent water repellency have been exploited either by mimicking the micro-/nanoscale textures of natural non-wetting surfaces or by coating with low free energy substances. [21-25] However, the practical exploitation of these superhydrophobic surfaces is severely hampered by their poor mechanical durability as well as susceptibility to oily contamination and chemical corrosion. [26, 27]

To enhance mechanical robustness, superhydrophobic coatings including inorganic particles $[28,29]$ (e.g., functionalized $\mathrm{SiO}_{2}$ ) or applying on substrates that inherently feature abrasion resistance (e.g., concretes, [30] elastomers [31] and textiles [5]) have been developed. However, the mechanical durability of such coatings is highly dependent on the adhesive strength of the applied coating layer, [32] and the superhydrophobicity cannot be preserved after the coating layer is worn off. Moreover, the coating layer normally suffers its instability from organic solvents, and oil fouling could negate its superhydrophobicity as well. [33]

Fabrication of water repellent bulk materials is a new concept in designing robust superhydrophobic material as the superhydrophobicity is found throughout the whole bulk volume and not just confined to its surface. To date, only a few studies have reported the exploitation of superhydrophobic free-standing items by simply compressing the composite particulates of inorganic nanofillers and polymers. For instance, Zhou and co-workers [34] reported a superhydrophobic free-standing composite by directly pressing the mixture of polytetrafluoroethylene particulates and carbon nanotubes. Despite of the improved damagetolerant superhydrophobicity for the obtained material, this method suffers from the cost and 
environmental issues due to the use of fluorinated compounds. To avoid the use of fluorides, Zhang and co-workers $[35,36]$ dispersed hydrophobic $\mathrm{SiO}_{2}$ nanoparticles in nonfluorinated polymer (e.g., polystyrene and polyhexamethylene adipamide) solutions, followed by precipitation and drying of the mixture to get composite particles, subsequently, the obtained particles were pressed under high pressure at room temperature to achieve superhydrophobic bricks. Although these superhydrophobic free-standing items exhibit a better mechanical durability compared to traditional superhydrophobic surfaces, they still feature poor stability, especially when they are exposed to organic solvents, due to the absence of a gluing layer between the particulates. Moreover, these superhydrophobic free-standing items still lose their superhydrophobicity after contaminated with oily dirt. Therefore, fabrication of superhydrophobic materials that simultaneously feature mechanochemical robustness and good resistance to oil fouling is extremely appealing but remains challenging.

In this work, we report all-organic fluorine-free superhydrophobic bulk material exhibiting photocatalytic functionality based on polypropylene (PP) and graphitic carbon nitride $\left(\mathrm{g}_{-} \mathrm{C}_{3} \mathrm{~N}_{4}\right)$. Beyond this, the material features mechanochemical robustness and good resistance to oil fouling. The as-prepared bulk material demonstrates excellent water repellency when exposed to either air or oil and shows good resistance to highly corrosive liquids (e.g., strong acids and bases) as well as organic solvents. Mechanical damage such as intensive abrasion, compression, tape-peeling or even cutting deeply into the material, do not lead to the loss of superhydrophobicity. Notably, the bulk material possesses photocatalytic self-cleaning functionality under visible light irradiation due to the included $\mathrm{g}-\mathrm{C}_{3} \mathrm{~N}_{4}$, which enables the elimination of oily dirt contaminated on the substrate surface, leading to the regeneration of its superhydrophobicity even after oil contamination. In addition, this strategy can be used as a general method to fabricate various kinds of superhydrophobic bulk materials with visiblelight-driven photocatalytic self-cleaning functionality. The exploitation of bulk materials with 
robust superhydrophobicity throughout its whole volume and simultaneously feature photocatalytic self-cleaning functionality paves the way on the design of energy-saving and maintenance-free self-cleaning materials that can be used in harsh chemical and oily environments.

\section{Experimental Section}

\subsection{Materials}

Toluene (99.8\%), tetrahydrofuran (THF) ( $\geq 99.5 \%), \mathrm{N}, \mathrm{N}$-dimethylformamide (DMF) $(\geq$ 99.8\%), chloroform ( $\geq 99 \%$ ), dicyandiamide (99\%), hydrochloric acid (37\%), sodium hydroxide ( $\geq 97 \%$ ), oleic acid ( $\geq 99 \%$ ) and rhodamine B ( $\geq 95 \%)$ were purchased from Sigmaaldrich. Ethanol (absolute for analysis) and acetone (absolute for analysis) were purchased from Merck Millipore. Polypropylene particulates of $\sim 10 \mu \mathrm{m}$ (PROPOLDER ${ }^{\mathrm{TM}}$ FPP4010) was supplied by TWOH Chem Ltd. PDMS (Sylgard 184, silicone elastomer base and curing agent) was purchased from Dow Corning. Milli-Q water was produced by a Millipore Simplicity system (Billerica, MA, USA). Unless otherwise mentioned all other chemicals were used as received.

\subsection{Sample preparation}

Graphitic carbon nitride $\left(\mathrm{g}-\mathrm{C}_{3} \mathrm{~N}_{4}\right)$ was synthesized by a thermal polycondensation method using dicyandiamide as a precursor. The reaction was conducted in a muffle furnace with the precursor in a ceramic crucible covered with the cap under the temperature of $550{ }^{\circ} \mathrm{C}$ for $2 \mathrm{~h}$ with the heating rate of $5{ }^{\circ} \mathrm{C} \cdot \mathrm{min}^{-1}$. The obtained $\mathrm{g}-\mathrm{C}_{3} \mathrm{~N}_{4}$ was further ground by a ball mill (Retsch PM100, Germany) at $500 \mathrm{rpm}$ for $2 \mathrm{~h}$. To prepare the bulk composites, $0.5 \mathrm{~g}$ g- $\mathrm{C}_{3} \mathrm{~N}_{4}$ and $5 \mathrm{~g}$ polymer (PP or PE) particulates were mixed with $1.5 \mathrm{~g}$ PDMS (silicone elastomer and curing agent at 10:1 by weight) and $5 \mathrm{ml}$ toluene, and the resultant mixture was mechanically stirred at $500 \mathrm{rpm}$ for $30 \mathrm{~min}$. Subsequently, the obtained blend was placed into a mold and 
pressed at $110^{\circ} \mathrm{C}$ using a template-based hot-press technique. The applied pressure and pressing time were $20 \mathrm{kPa}$ and 20 minutes, respectively. The obtained bulk composites were treated at $80{ }^{\circ} \mathrm{C}$ under vacuum condition for $12 \mathrm{~h}$ before any further characterization.

\subsection{Characterizations}

A high resolution scanning electron microscope (SEM) combined with Energy Dispersive XRay (EDX) (Zeiss Supra $50 \mathrm{VP}$, Germany) were used to characterize the bulk composite material microstructures. The typically used electron acceleration voltage was $10 \mathrm{keV}$. Prior to SEM-EDX analysis the samples were coated with $10 \mathrm{~nm}$ layer of platinum via a sputter coater (Safematic CCU-010, Switzerland). Fourier transform infrared (FTIR) spectra were obtained with a Bruker vertex 70 attenuated total reflection (ATR) FTIR Spectrometer equipped with an ATR single reflection crystal (Bruker Optic GmbH, Germany). The spectra were collected in the range of $400-4000 \mathrm{~cm}^{-1}$ (64 scans) and the background spectra were recorded against air. Powder X-ray diffraction (XRD) analysis was performed by a PANalytical X'pert diffractometer operated at $40 \mathrm{kV}$ and $40 \mathrm{~mA}$ using $\mathrm{Cu}$ Ka radiation. Photoluminescence (PL) measurements were recorded on a Hitachi F-7000 fluorescence spectrometer. UV-vis spectroscopy was measured on a Shimadzu UV-2600 spectrophotometer. An RPR-200 model reactor (SNE Ultraviolet Co., USA) with eight UV lamps (SNE Ultraviolet Co., USA) featuring emission wavelength at $350 \mathrm{~nm}$ was employed to evaluate the UV resistance property of the asprepared bulk material. The contact angle and sliding angle measurements were performed using a Contact Angle System OCA (Stuttgart, Germany).

Mechanical performance tests. The measurements were performed with an Instron 3345 universal testing device. For the flexural tests, a three-point bending setup was used. The support span was set to be $18 \mathrm{~mm}$. The typical sample dimensions were $30 \mathrm{~mm} \times 10 \mathrm{~mm} \times 1$ $\mathrm{mm}$. A gap of $30 \mathrm{~mm}$ was used in the tensile measurements and the typical sample dimensions were $60 \mathrm{~mm} \times 10 \mathrm{~mm} \times 1 \mathrm{~mm}$. In compressive measurements, circular samples with diameter 
of $15 \mathrm{~mm}$ and height of $5 \mathrm{~mm}$ were used. The applied testing rate for all the measurements was $1 \mathrm{~mm} \cdot \mathrm{min}^{-1}$. For average values of the maximum stress and strain, at least three specimens of each type sample were measured.

Abrasion test. The mechanical abrasion measurements were conducted using a reciprocating sliding abrasion machine (TQC, Germany). The friction partner (280 grit sand paper) was mounted on a reciprocating sled which oscillates with a given stroke speed. Each reciprocating sliding abrasion was counted as one abrasion cycle. During abrasion test, the samples were mounted on the sample holders with double sided tape. The applied load, stroke speed and distance were $530 \mathrm{~g}, 15$ cycles $\cdot \mathrm{min}^{-1}$ and $30 \mathrm{~cm}$, respectively. The contact angles and sliding angles of the abraded samples were measured as a function of abrasion cycles.

Tape-peeling test. An adhesive tape (3M, Scotch® Magic ${ }^{\mathrm{TM}}$ Tape 810$)$ with adhesion force of $250 \mathrm{~N} \cdot \mathrm{m}^{-1}$ to steel (ASTM D-3330) was used for the tape-peeling test. The tape was placed and pressed with a load of $900 \mathrm{~g}$ on the superhydrophobic bulk composite surface to achieve uniform contact between the tape and the substrate, followed by slowly peeling the tape away from the substrate surface. The contact angles and sliding angles of the tape-peeled substrates were investigated as a function of tape-peeling cycles.

Photocatalytic experiments. All the photocatalytic experiments were carried out by using a 300W Xe lamp as the light resource (light intensity of $40.8 \mathrm{~mW} \cdot \mathrm{cm}^{-2}$ ) with a visible light filter (> $420 \mathrm{~nm}$ ). The bulk material surface was contaminated by adhesion of oleic acid $\left(\mathrm{CH}_{3}\left(\mathrm{CH}_{2}\right)_{7} \mathrm{CH}=\mathrm{CH}\left(\mathrm{CH}_{2}\right)_{7} \mathrm{COOH}\right)\left(\right.$ dissolved in acetone, $\left.0.5 \mathrm{~mol} \cdot \mathrm{L}^{-1}\right)$. Visible light irradiation was carried out for $6 \mathrm{~h}$ to decompose the oleic acid contaminated on the bulk material surface. The photocatalytic performance was also evaluated by the decolorization of rhodamine $\mathrm{B}$ (dissolved in ethanol, $10 \mathrm{mg} \cdot \mathrm{L}^{-1}$ ) contaminated on the bulk composite material surface, and the visible light irradiation was performed for $2 \mathrm{~h}$. 


\section{Results and Discussion}

\subsection{Achieving the Functional Bulk Material}

Prior to the bulk material preparation, $\mathrm{g}-\mathrm{C}_{3} \mathrm{~N}_{4}$ was synthesized using dicyandiamide as a precursor (Fig. 1a). The crystallographic structure of the obtained $g-\mathrm{C}_{3} \mathrm{~N}_{4}$ was characterized with X-ray diffraction (XRD) technique, as shown in Fig. 1b. The intense peaks at $2 \theta$ value of $\sim 27^{\circ}$ and $\sim 13^{\circ}$ indexing to the (002) and (100) crystal planes of $\mathrm{g}-\mathrm{C}_{3} \mathrm{~N}_{4}$, which are ascribed to the in-plane structural packing motif and the inter-planar stacking of graphite-like conjugated triazine aromatic sheets, respectively. [37] TEM was also used to investigate the morphology and microstructure of the obtained $\mathrm{g}-\mathrm{C}_{3} \mathrm{~N}_{4}$, and the result is shown in Fig. 1c.

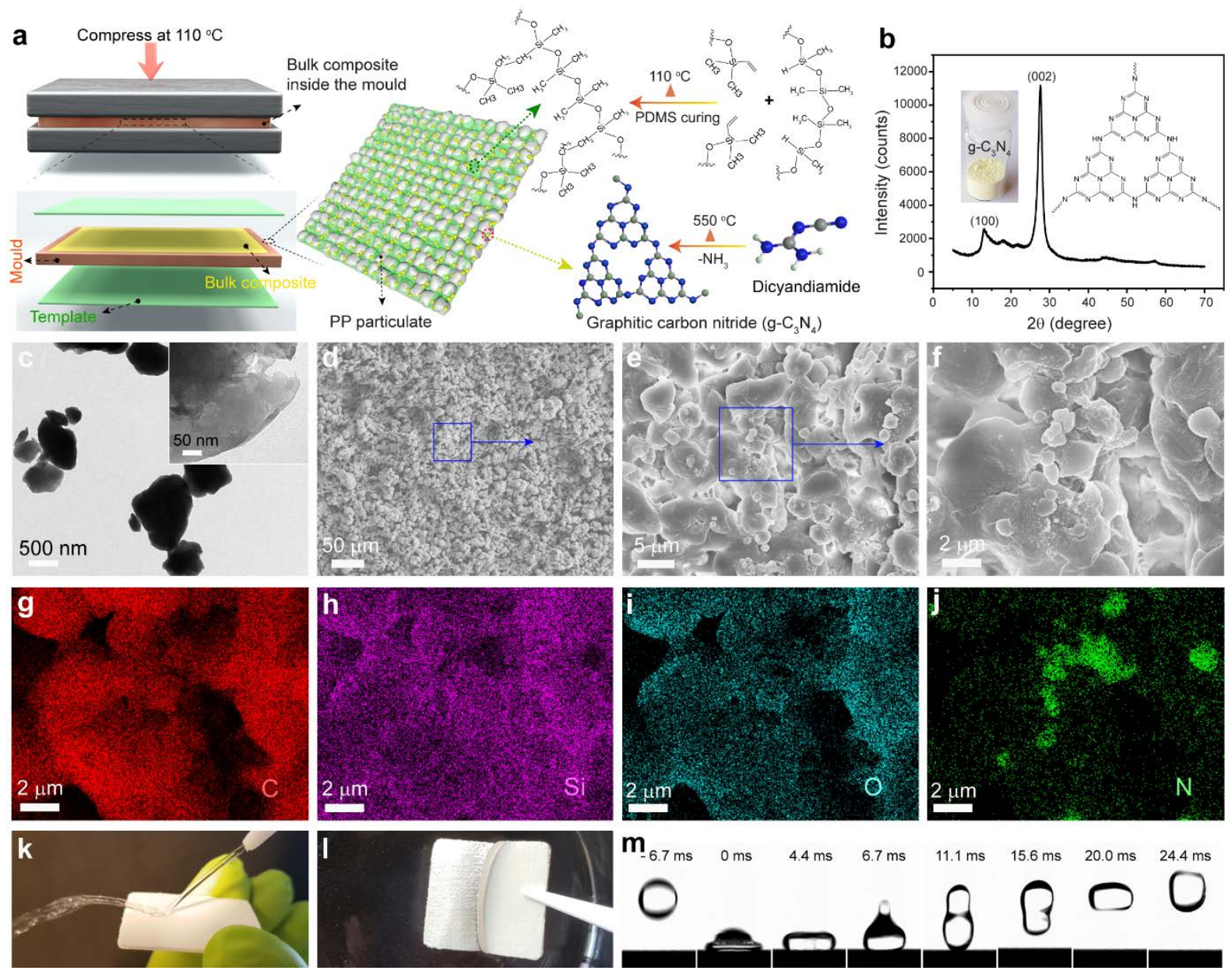

Fig. 1. (a) Strategy of the bulk composite material preparation. The curing of PDMS was induced during the pressing procedure at $110^{\circ} \mathrm{C} . \mathrm{g}-\mathrm{C}_{3} \mathrm{~N}_{4}$ was synthesized from dicyandiamide at $550{ }^{\circ} \mathrm{C}$ prior to the fabrication of the bulk composites. (b) X-ray diffraction (XRD) patterns 
of $\mathrm{g}-\mathrm{C}_{3} \mathrm{~N}_{4}$. The insets show a photo of the synthesized $\mathrm{g}-\mathrm{C}_{3} \mathrm{~N}_{4}$ (left) and its chemical formula (right). (c) TEM images of the synthesized $g-\mathrm{C}_{3} \mathrm{~N}_{4}$. (d-f) SEM images showing the microscopic structure of the bulk composite at different magnifications. (g-j) Corresponding EDX mapping of the bulk composite. Photos showing the bulk composite with $(\mathrm{k})$ a jet of water bouncing off (with jet flow of $158 \mathrm{ml} \cdot \mathrm{cm}^{-2} \cdot \mathrm{s}^{-1}$ and incident angle of $\sim 45^{\circ}$ with respect to the bulk composite surface) and (1) mirror-like phenomenon in water under natural light (observation angle of $50^{\circ}$ with respect to the bulk composite surface). (m) Time resolved images showing a $10 \mu \mathrm{L}$ water droplet bouncing on the bulk composite surface.

The as-prepared $\mathrm{g}-\mathrm{C}_{3} \mathrm{~N}_{4}$ was subsequently employed as photocatalyst to blend with $\mathrm{PP}$ particulates and PDMS (i.e., siloxane oligomer and siloxane cross-linkers), followed by compressing the mixture into bulk composites using a template-based hot-press technique (Fig. 1a). Commercially available 400 grit sandpapers (Fig. S1) were used as templates to retain the rough surface morphology. During hot pressing, the cross-linking between siloxane oligomer and siloxane cross-linkers was induced (Fig. 1a), [38] resulting in the formation of PDMS elastomer, which acts as the binder between $\mathrm{PP}$ and $\mathrm{g}-\mathrm{C}_{3} \mathrm{~N}_{4}$ particulates and promote the robustness of the obtained bulk composites.

Fig. 1d-f shows the scanning electron microscopy (SEM) images of the microstructures for the obtained bulk composites. A hierarchical rough morphology can be clearly observed: the rough surface morphology resulted from template-based hot pressing (with protrusion height of $\sim 55 \mu \mathrm{m}$, as shown in Fig. S1a-b); the stacking PP particles $(\sim 10 \mu \mathrm{m})$ agglutinated by crosslinked PDMS are clearly visible as well as the $\mathrm{g}-\mathrm{C}_{3} \mathrm{~N}_{4}$ particulates $(<2 \mu \mathrm{m})$ attached on the PP surface. The highly dispersed $\mathrm{g}-\mathrm{C}_{3} \mathrm{~N}_{4}$ particulates in the bulk composite was further confirmed by the presence of corresponding nitrogen signal from energy dispersive X-ray spectroscopy (EDX) results (Fig. 1g-j). 
The obtained bulk composite features excellent water repellency. As shown in Fig. 1k, a jet of water can easily bounce off the bulk composite surface without leaving any trace (Movie 1, Supporting Information). A mirror-like reflective behavior was observed when the bulk material was submerged in water (Fig. 11) and it remained completely non-wetting after being taken out, which directly demonstrated the existence of the air cushion between the solid surface and water. [39] This is in accordance with the Cassie-Baxter theory; hence, the interaction between the solid surface and water is very weak, resulting in the superhydrophobicity of the as-prepared bulk composites. Strikingly, when a water droplet fell on the bulk composite, it can easily and completely bounce off from the solid surface instead of resting on it (Fig. 1m and Movie 2 (Supporting Information)). These observations indicate the ultra-low adhesion of the solid surface with water and well explain the very high water contact angle $\left(\theta_{C A}\right)\left(\sim 171.0^{\circ}\right)$ and very low sliding angle $\left(\theta_{S A}\right)\left(\sim 7.0^{\circ}\right)$ of the obtained material. These unique performances of the bulk composite can be attributed to its surface microscale roughness along with the hydrophobicity of PP particulates and the low surface energy of the binding PDMS. The nonwetting behavior of the water droplets on the bulk composite surface can be elaborated by the Cassie model. [40]

\subsection{Mechanochemical Robustness}

Mechanical susceptibility is the main issue that limits the practical application of superhydrophobic surfaces. Here, four different types of mechanical robustness tests were conducted for the as-prepared bulk composites and the results are shown in Fig. 2.

A schematic of the abrasion test is presented in Fig. 2a. The changes in $\theta_{C A}$ and $\theta_{S A}$ of the bulk composite with the abrasion cycles are shown in Fig. 2b. The $\theta_{C A}$ of the bulk composite remained around $170^{\circ}$ after 150 reciprocating abrasion cycles. $\theta_{S A}$ was slightly increased after 150 cycles abrasion, yet it remained under $10^{\circ}$. This indicates an excellent abrasion-resistant superhydrophobicity of the as-prepared bulk composite. We attribute the above abrasion- 
durable superhydrophobicity to the microstructure of the bulk composite throughout its whole volume as well as the micro- and nanoscale rough surface texture induced by the abrasion (Fig. 3a).

To demonstrate the bulk's superhydrophobic properties $\theta_{C A}$ and $\theta_{S A}$ measurements have also been performed on freshly generated surfaces of the bulk material by cutting the solid body. Here, cylindrical bulk composites $(10 \mathrm{~mm}$ in height and $5 \mathrm{~mm}$ in diameter) were prepared, followed by cutting deeply into it at different distance (Fig. 2a). The water repellency of the cut cross-sections was measured. The freshly cut cross-sections exhibit comparable $\theta_{C A}$ of $\sim 170^{\circ}$ and $\theta_{S A}$ of $\sim 9^{\circ}$ (Fig. 2c), which indicates that the internal part of the bulk composites features superhydrophobic properties. This is further verified by a spherical water droplet sitting on the cross-section crack of the bulk composite (Fig. 2f). The above observations suggest that the obtained bulk composite material exhibit excellent water repellency extending throughout its whole volume. This is evidently explained by the micro-/nanoscale roughness features of the cut and randomly fractured surface, as shown in Fig. 3b and c, respectively. 

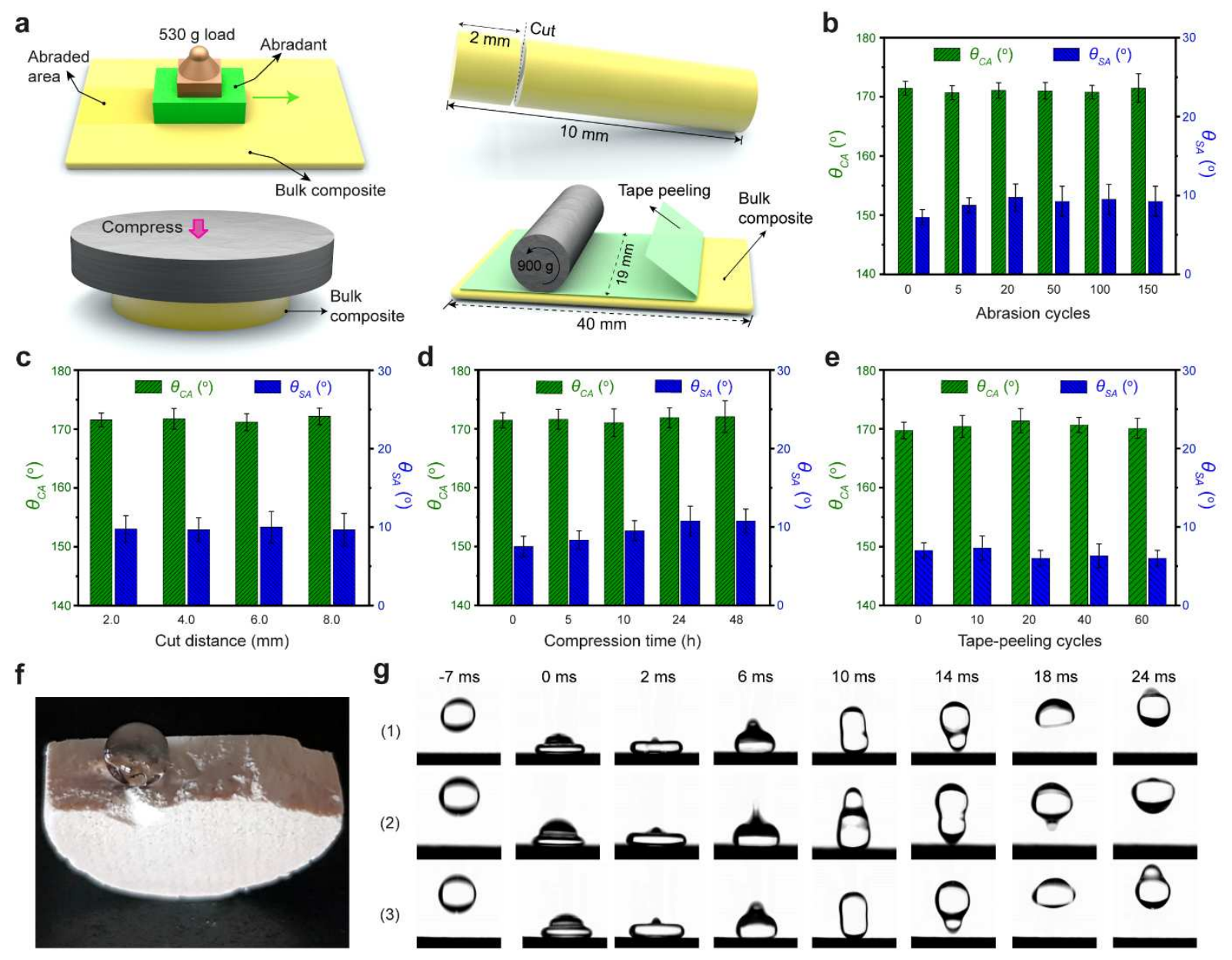

Fig. 2. (a) Schematics of abrasion, cutting, compression and tape-peeling tests. The effect of (b) abrasion cycles, (c) cutting position from surface, (d) compression time and (e) tape-peeling cycles on the $\theta_{C A}$ and $\theta_{S A}$ of the prepared bulk composites. (f) A $10 \mu \mathrm{L}$ water droplet spherically sitting on the cross-sectional crack showing superhydrophobicity inside the bulk composite. (g) The complete bouncing of a $10 \mu \mathrm{L}$ water droplet on the bulk composite surface (1) after 150 cycles abrasion at $530 \mathrm{~g}$ load, (2) after $48 \mathrm{~h}$ compression under $14 \mathrm{kPa}$ and (3) after 60 cycles of tape-peeling.

The water repellency of the as-prepared bulk material exhibits highly compression and tapepeeling resistant, as well. The schematics of compression and tape-peeling tests are shown in Fig. 2a. After $48 \mathrm{~h}$ compression at $14 \mathrm{kPa}, \Theta_{C A}$ remained around $170^{\circ}$, whereas a slight increase of $\theta_{S A}$ from $\sim 7^{\circ}$ to $\sim 11^{\circ}$ was observed (Fig. 2d). The retained superhydrophobicity of the bulk composite after compression is ascribed to its remaining rough surface texture (Fig. 3d). Fig. 
2e shows that even after 60 cycles of tape-peeling the $\theta_{C A}$ and $\theta_{S A}$ remains $\sim 170^{\circ}$ and $\sim 7^{\circ}$, respectively. The complete bounce of a water droplet on the abraded, compressed or tape-peeled bulk composite surface (Fig. 2g) further confirms the retained superhydrophobicity. We attribute the above mechanically robust superhydrophobicity of our bulk composite to the micro-/nanostructures throughout its whole volume along with the introduced low surface energy PDMS binders.

The mechanical properties, e.g. flexural, tensile and compressive properties, are vital features of bulk materials for practical applications, yet they have never been reported for a superhydrophobic bulk material. Here, the mechanical performance of the obtained superhydrophobic bulk composites were investigated, as well. Flexural tests were performed on the bulk composite to determine its bending properties (Fig. 3e). The flexural strength, strain and modulus for the as-prepared bulk composites were determined to be $16.9 \pm 1.41 \mathrm{MPa},(2.5$ $\pm 0.53) \%$ and $1.5 \pm 0.26 \mathrm{GPa}$, respectively. In addition, the tensile and compressive measurements were also conducted on such bulk composites (Fig. $3 \mathrm{f}$ and g). The bulk composites featuring the tensile strength and strain of $6.0 \pm 0.49 \mathrm{MPa}$ and $(2.7 \pm 0.44) \%$, respectively, and it can survive with a compressive stress of up to $27.1 \pm 1.92 \mathrm{MPa}$ before being crushed. These results indicate good mechanical properties of the obtained bulk material. For comparison, composite materials (PP/PDMS) without g- $\mathrm{C}_{3} \mathrm{~N}_{4}$ and composites $\left(\mathrm{PP} / \mathrm{g}-\mathrm{C}_{3} \mathrm{~N}_{4}\right)$ containing no PDMS were prepared and evaluated with tensile measurements (Fig. S2). When compared with the as-prepared bulk material, comparable tensile strength was obtained for the composites containing same amount ratio of PDMS but without $\mathrm{g}-\mathrm{C}_{3} \mathrm{~N}_{4}$, whereas a much lower tensile strength was found for the composites containing no PDMS. These observations suggest that the introduced PDMS binder promotes the mechanical robustness of the bulk material. 


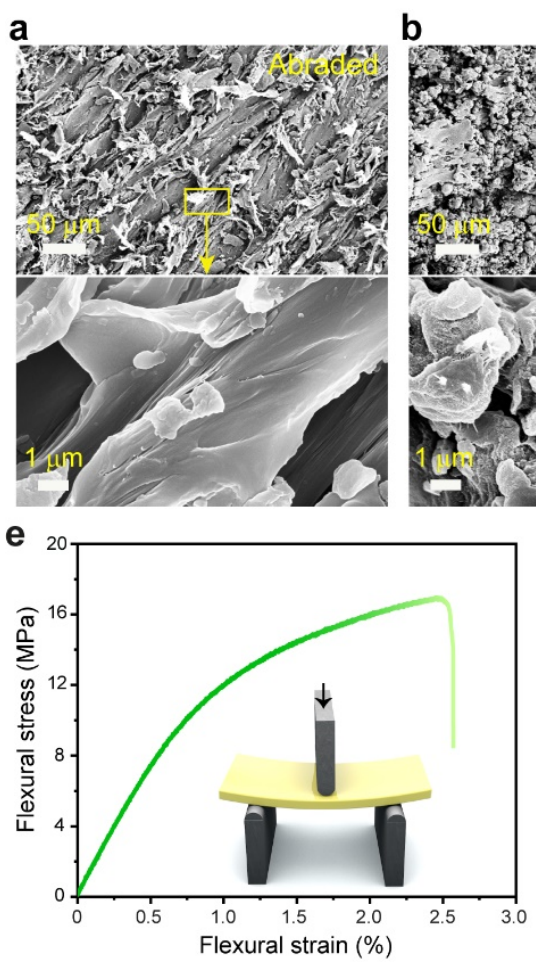

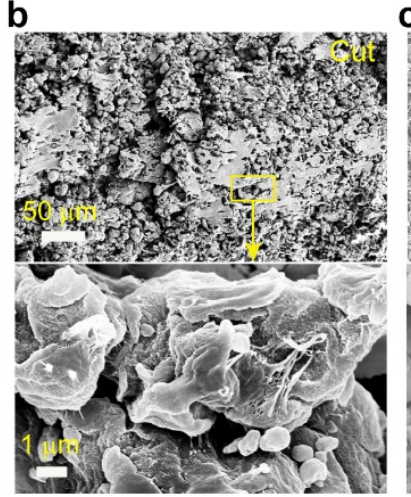

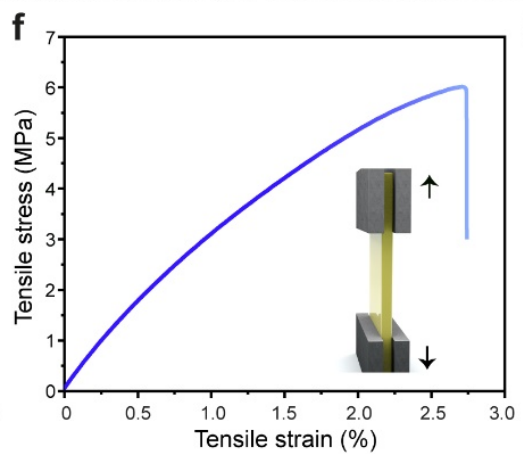

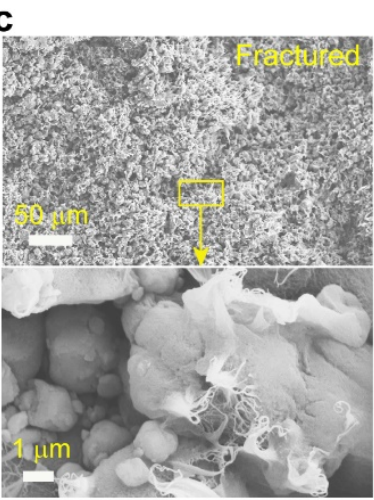
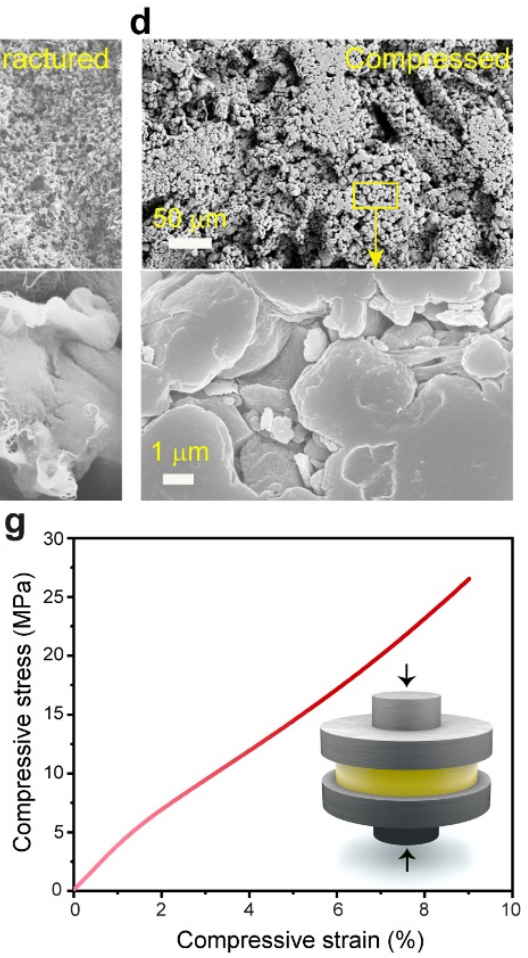

Fig. 3. SEM images of (a) the abraded surface after 150 cycles abrasion, (b) freshly cut section,

(c) randomly fractured surface and (d) compressed surface under14 kPa for $48 \mathrm{~h}$. (e) Flexural,

(f) tensile and (g) compressive test results of the bulk composites. The inserts showing the corresponsive schemes of the mechanical performance tests for the bulk composites.

Chemical durability plays a vital role in the practical application of superhydrophobic materials, as well. [33] Here, chemical durability of the prepared bulk composites was assessed by immersing the bulk material in the corrosive solutions and periodically removing the samples, followed by measuring the $\theta_{C A}$ and $\theta_{S A}$ of the corroded samples after water rinsing and drying.

$0.1 \mathrm{M}$ hydrochloric acid $(\mathrm{HCl})$ and $0.1 \mathrm{M}$ sodium hydroxide $(\mathrm{NaOH})$ solutions were used to assess the chemical corrosion resistance of the bulk composite. Fig. $4 \mathrm{a}$ and b present the effect of corrosion time on $\theta_{C A}$ and $\theta_{S A}$ of the bulk composites in $\mathrm{HCl}$ and $\mathrm{NaOH}$ solutions, respectively. A gradual decrease of $\theta_{C A}$ and increase of $\theta_{S A}$ were observed with increase of corrosion time in both $\mathrm{HCl}$ and $\mathrm{NaOH}$ solutions. However, after $1 \mathrm{~h}$ corrosion from both $\mathrm{HCl}$ and $\mathrm{NaOH}$ solutions, the $\theta_{C A}$ and $\theta_{S A}$ of the bulk composites remained above $165^{\circ}$ and below 
$20^{\circ}$, respectively. SEM images of the bulk composites surface show no observable damage after both $\mathrm{HCl}$ (Fig. 4c) and $\mathrm{NaOH}$ (Fig. 4d) corrosion. Strikingly, after the corroded surface was removed by mechanical abrasion, the original water repellency of the bulk material can be restored due to the exposure of renewed surfaces (Fig. 4a and b). Note that this restoration procedure can be continuously repeated until the bulk composites have been worn out.
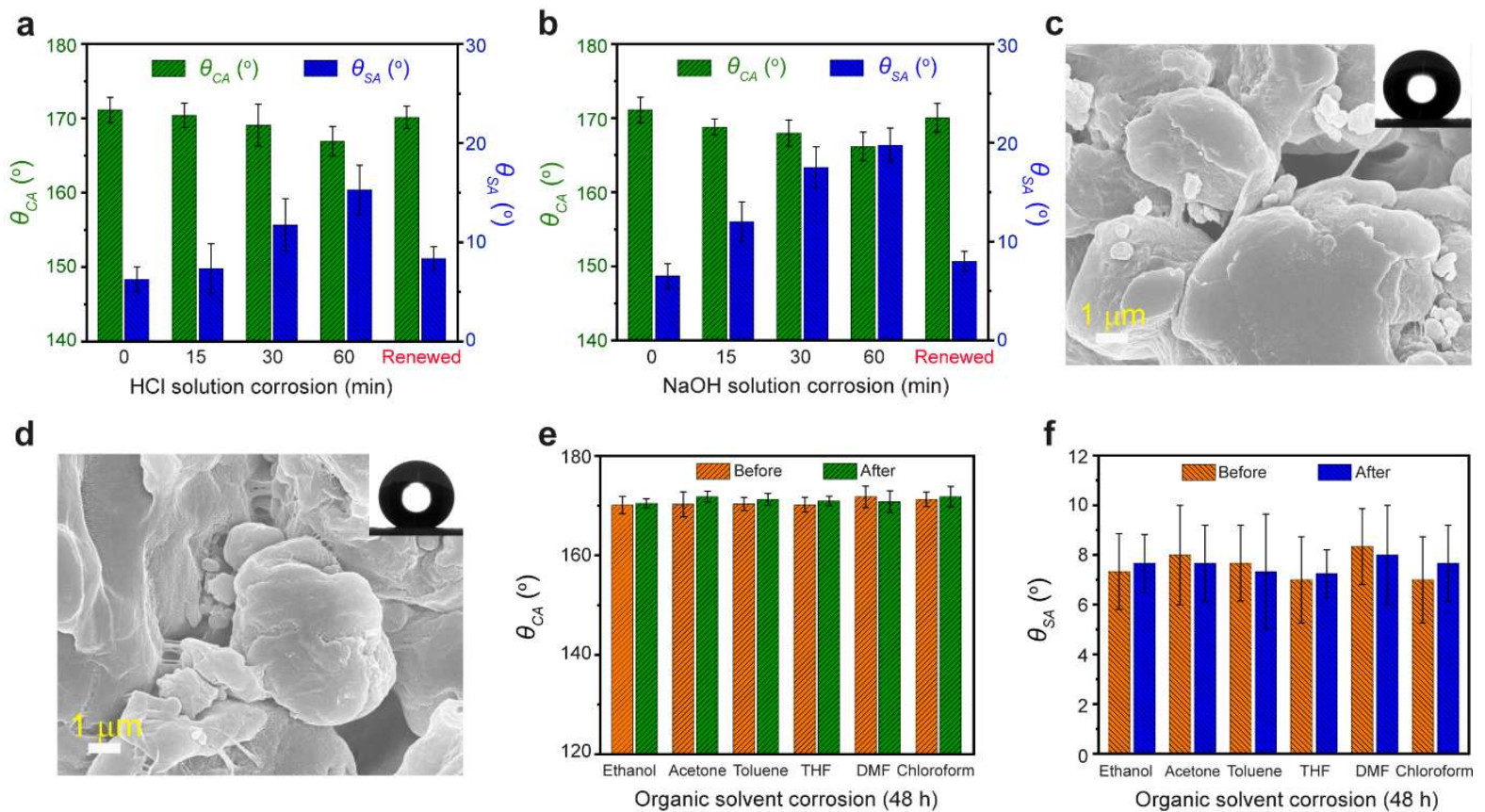

Fig. 4. Influence of (a) $\mathrm{HCl}$ and (b) $\mathrm{NaOH}$ solution corrosion time on the water repellency of the bulk composite. SEM micrograph of the bulk material surface after 60 min corrosion in (c) $\mathrm{HCl}$ and (d) $\mathrm{NaOH}$ solutions. (e) $\theta_{C A}$ and (f) $\theta_{S A}$ of the bulk composites before and after $48 \mathrm{~h}$ of corrosion in different organic solvents.

Moreover, the superhydrophobic bulk composites demonstrate excellent resistance towards organic solvents, as well. The $\theta_{C A}$ and $\theta_{S A}$ of the bulk material before and after $48 \mathrm{~h}$ submersion in the test organic solvents were measured. In contrast to the reported superhydrophobic coatings [33, 41] and free-standing items [35-36], our bulk composites exhibit excellent stability towards these aggressive solvents, such as dimethylformamide (DMF), tetrahydrofuran (THF) and chloroform, due to the induced crosslinked PDMS binder 
layer between the particulates. After $48 \mathrm{~h}$ submersion, both the $\theta_{C A}$ and $\theta_{S A}$ of the bulk composites surface remained changeless for all the tested organic solvents (Fig. 4e and f), and the exposed surface exhibited no observable damage (Fig. S3, Supporting Information). In addition, the obtained superhydrophobic bulk material features good resistance towards UV irradiation, for instance, after $12 \mathrm{~h}$ exposure to UV light, no observable damage was found for the microrough surface and the $\theta_{C A}$ and $\theta_{S A}$ of the bulk material were maintained around $170^{\circ}$ and below $10^{\circ}$, respectively (Fig. S4, Supporting Information).

These observations reveal that our bulk materials feature good chemical corrosion resistance and excellent restorative ability of water repellency compared to widely reported superhydrophobic surfaces. Such excellent anticorrosive repellency can be attributed to the synergistic effect of the high chemical stability of the bulk composite and the micro-rough structures throughout its whole volume.

\subsection{Photocatalytic Self-cleaning Functionality}

Superhydrophobic surfaces normally dispel their superhydrophobicity after contamination with oily dirt. However, the as-prepared bulk composite features the ability of regeneration of superhydrophobicity even after oily dirt contamination due to its photocatalytic self-cleaning functionality. $[42,43]$ To investigate the photocatalytic self-cleaning properties of the obtained bulk material, we contaminated the sample with oleic acid as a model of oily dirt. The regeneration of superhydrophobicity was examined by measuring the changes in $\theta_{C A}$ and $\theta_{S A}$ of the contaminated bulk composite before and after visible light irradiation. As shown in Fig. 5a-c, after contamination with oleic acid $\theta_{C A}$ of the bulk material was decreased from $\sim 171^{\circ}$ to $\sim 114^{\circ}$, and the water droplets on its surface cannot roll off $\left(\Theta_{S A}>90^{\circ}\right)$. After $6 \mathrm{~h}$ visible light irradiation, the superhydrophobicity of the bulk composite (containing $g-\mathrm{C}_{3} \mathrm{~N}_{4}$ ) was recovered (Fig. 5a-c) due to its photocatalytic self-cleaning functionality which resulted from the photocatalytic activity of the included $\mathrm{g}_{-} \mathrm{C}_{3} \mathrm{~N}_{4}$. In contrast, the bulk material without $\mathrm{g}$ - 
$\mathrm{C}_{3} \mathrm{~N}_{4}$ could not recover its superhydrophobic state even after visible light irradiation (Fig. S5, Supporting Information). This shows that the removal of oleic acid on the bulk material surface was realized by the photocatalytic activity of the included $g-\mathrm{C}_{3} \mathrm{~N}_{4}$. Furthermore, after 5 cycles of contamination and successive photodegradation of oleic acid, the bulk composite (with g$\mathrm{C}_{3} \mathrm{~N}_{4}$ ) maintains its water-repellent sate with $\theta_{C A}$ of $\sim 170^{\circ}$ and $\theta_{S A}$ of $\sim 7^{\circ}$, as shown in Fig. $5 \mathrm{~b}$ and $\mathrm{c}$. The photocatalytic performance of the bulk material on the degradation of oleic acid was revealed by FTIR absorbance spectra, as well (Fig. 5d). The presence of absorbance band at $1709 \mathrm{~cm}^{-1}$ following oleic acid contamination can be clearly observed in the FTIR spectra, which is ascribed to the $\mathrm{C}-\mathrm{O}$ stretching of oleic acid. [44] This absorbance band disappeared after $6 \mathrm{~h}$ of visible light irradiation, which indicates quantitative photodegradation of oleic acid.

The photodegradation of organic molecules is not limited to the oily contamination. To further investigate the self-cleaning property, we contaminated the bulk composite material with both inorganic micro-sized particles (quartz sand) and an organic dye (rhodamine B, indicated by the pink color on the substrate surface, Fig. 5e (1)). As a comparison, bulk material without $\mathrm{g}-\mathrm{C}_{3} \mathrm{~N}_{4}$ was contaminated with the inorganic particles and dye as well (Fig. 5e (2)). After rinsing with water, the micro-sized particles were efficiently removed from the bulk material surface (Fig. 5e), which is due to the lotus effect of the water repellent surfaces [45] that the particles were taken along by the rolling water drops (Movie 3, Supporting Information). Although inorganic particles can be efficiently removed by water rinsing, the organic contaminant remaining on the surface are typically difficult to remove from a superhydrophobic surface without washing by organic solvents (Fig. 5e). To remove the remained organic dye, the bulk materials were subsequently irradiated with visible light. After $2 \mathrm{~h}$ of visible light irradiation, the pink color disappeared from the surface of the bulk material containing $\mathrm{g}_{-} \mathrm{C}_{3} \mathrm{~N}_{4}$ (Fig. 5e (1)), while the one without $g-\mathrm{C}_{3} \mathrm{~N}_{4}$ remains pink (Fig. 5e (2)). The decolorization of the bulk material with $\mathrm{g}-\mathrm{C}_{3} \mathrm{~N}_{4}$ indicates that the degradation of organic dye was achieved by 
the photocatalytic activity of the included $\mathrm{g}_{-} \mathrm{C}_{3} \mathrm{~N}_{4}$. Strikingly, even after 10 cycles of dye contamination and successive visible light irradiation, the superhydrophobicity of the bulk material remains unchanged (Fig. S6, Supporting Information).

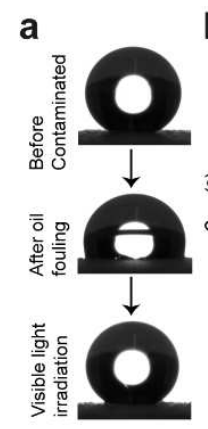

b

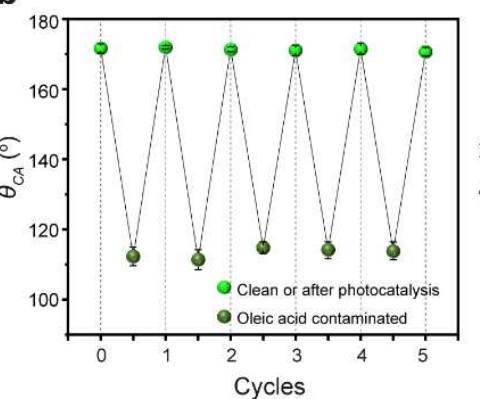

c

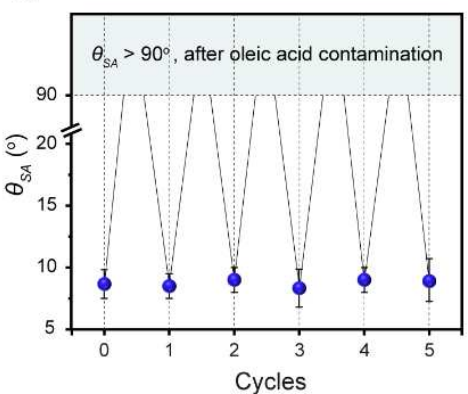

d

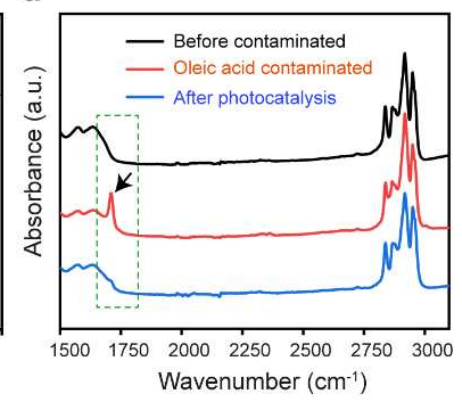

e organic

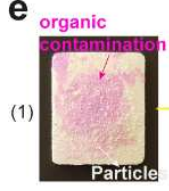

Contaminated

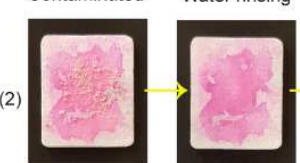

$\mathbf{f}$

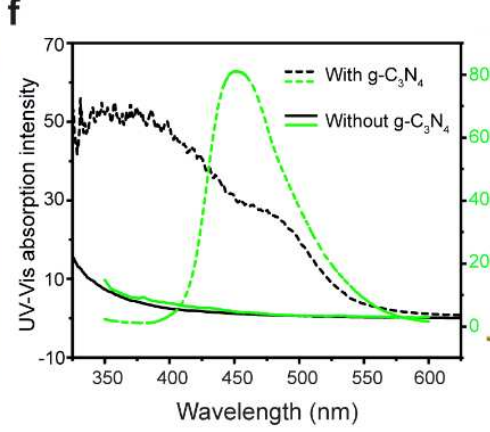

g

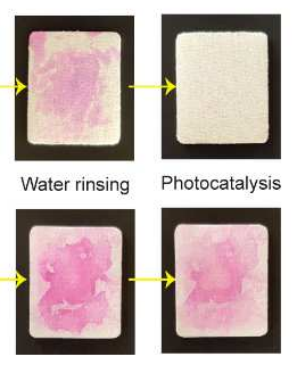

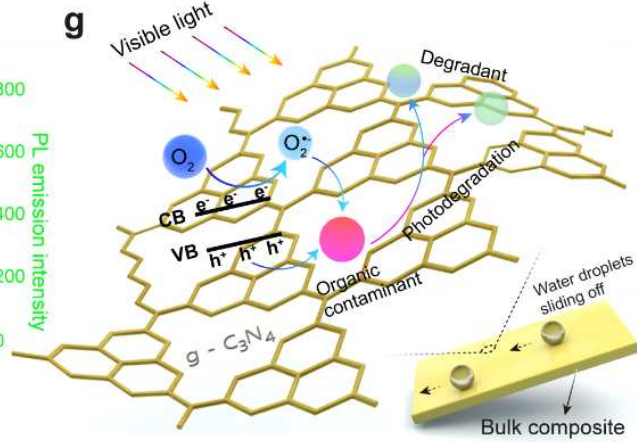

Fig. 5. (a) Images of a water droplet on the bulk composite (with $g-\mathrm{C}_{3} \mathrm{~N}_{4}$ ) surface before and after contamination with oleic acid, as well as after irradiation of visible light for $6 \mathrm{~h}$. Variation of the (b) $\theta_{C A}$ and (c) $\theta_{S A}$ of the bulk composite (with $\mathrm{g}-\mathrm{C}_{3} \mathrm{~N}_{4}$ ) in five cycles of oleic acid contamination and visible light irradiation. (d) Single reflection ATR-FTIR absorbance spectra of the bulk composite before and after oleic acid contamination, as well as after visible light irradiation for $6 \mathrm{~h}$. The black arrow in the FTIR spectra indicate the characteristic FTIR absorbance of oleic acid. (e) Images of self-cleaning process on the superhydrophobic bulk material. The bulk material surface was contaminated with rhodamine $\mathrm{B}(\mathrm{RhB})$ dissolved in ethanol and quartz sand particles. (f) The UV-Vis diffused reflectance spectra and photoluminescence (PL) spectra of the bulk composites with and without $\mathrm{g}_{-} \mathrm{C}_{3} \mathrm{~N}_{4}$. (g) Mechanism illustration for the photodegradation of organic contaminants on the superhydrophobic bulk composite. 
To prove the origin of the photocatalytic activity of the bulk composite material, UV-Vis absorption spectra and photoluminescence (PL) spectra were investigated further. As shown in Fig. 5f, the typical absorption edge appeared in the bulk composite with $\mathrm{g}-\mathrm{C}_{3} \mathrm{~N}_{4}$, while the one without $\mathrm{g}-\mathrm{C}_{3} \mathrm{~N}_{4}$ shows no response to the $\mathrm{UV}$-vis absorption, indicating the addition of $\mathrm{g}_{-} \mathrm{C}_{3} \mathrm{~N}_{4}$ endows photocatalytic capacity to the composite material. The PL spectra further confirmed the range of the light response for the bulk composite with $\mathrm{g}_{-} \mathrm{C}_{3} \mathrm{~N}_{4}$ is located in the visible area (400-550 nm), which agrees well with the reported bare $\mathrm{g}-\mathrm{C}_{3} \mathrm{~N}_{4}$ photocatalyst. [46] However, for the bulk composite containing no $\mathrm{g}-\mathrm{C}_{3} \mathrm{~N}_{4}$, there is no obvious absorption signal across the whole spectrum. Based on the above discussion and the typical photocatalytic process, we propose the photocatalytic reaction mechanism (Fig. 5g) in the following processes: [47-50] The visible light illuminates on $\mathrm{g}-\mathrm{C}_{3} \mathrm{~N}_{4}$ and stimulates the valence band (VB) to generate photoelectrons, which can easily go to the conduction band (CB) of $\mathrm{g}_{-} \mathrm{C}_{3} \mathrm{~N}_{4}$ and combine with the contacted $\mathrm{O}_{2}$ to form active $\mathrm{O}_{2}{ }^{*}$. The left holes in the valence band (VB) along with the $\mathrm{O}_{2}{ }^{\bullet}$ are the main active species for the photodegradation of oily contaminations and organic dyes.

Notably, the self-cleaning of our bulk material was found to function in either oil or air, as shown in Fig. S7 (Supporting Information). When the bulk material was immersed in oil, the oil can gradually penetrate through its surface, and the water droplets were supported by both oil and the surface microstructure. [51] This results in the similar self-cleaning performance in oil to that in air, [52] i.e., the bulk material maintained its water repellency and self-cleaning ability when immersed in oil (Fig. S7e-g). In air, after oil immersion, the surface microstructure of the bulk composite can lock the oil contamination as a lubricating fluid, resulting in a slippery state (Fig. S7d and Movie 4, Supporting Information). [53, 54] Dirt can be taken away from the bulk material surface by the sliding over water drops (Movie 5, Supporting Information).

In addition, with this strategy, superhydrophobic bulk composites based on polyethylene, and polystyrene particulates were prepared as well. Similar to the PP based bulk composite as 
discussed above, these bulk materials also feature excellent superhydrophobicity and photocatalytic self-cleaning functionality (see Fig. S8). The outstanding water repellency of the as-prepared bulk composites can be attributed to the microrough structures throughout their whole volume along with the low surface energy of the binding PDMS, while the photocatalytic self-cleaning performance is ascribed to the photocatalytic activity of the introduced $g-\mathrm{C}_{3} \mathrm{~N}_{4}$. Thus, the developed strategy can be used as a general hot press method to prepare robust superhydrophobic bulk materials with visible-light-driven photocatalytic self-cleaning functionality using $\mathrm{g}-\mathrm{C}_{3} \mathrm{~N}_{4}$ as the photocatalysis and PDMS as the glue binder between the particulates.

\section{Conclusion}

In summary, we presented a simple method to fabricate robust all-organic fluorine-free superhydrophobic bulk material that maintained water repellency under a variety of harsh mechanical and chemical environments as well as oil-fouling. Moreover, the bulk composite material possesses photocatalytic self-cleaning functionality due to the photocatalytic activity of the included $\mathrm{g}-\mathrm{C}_{3} \mathrm{~N}_{4}$, which enables the oil-fouled surface to regenerate its superhydrophobicity after visible light irradiation. With this work we were able to show the possibility to synthesize bulk materials combining superhydrophobic properties across the whole 3D material with the chemical and mechanical properties required for many applications. In addition, we were able to add photocatalytic self-cleaning properties. Applying this strategy, i.e. developing bulk materials with inherent superhydrophobic and/or self-cleaning properties, a major obstacle of superhydrophobic coatings can be circumvented. The robust and multifunctional bulk material developed here paves the way on the design of energy-saving and maintenance-free self-cleaning materials that can be used in various industry and daily life. 


\section{Conflicts of interest}

The authors declare that there is no conflict of interest.

\section{Acknowledgements}

The authors acknowledge the Center for Microscopy and Image Analysis (ZMB) of University of Zurich for the access to the microscopic facilities. We also thank Davide Bottone for the support of the high speed camera videos and Ethan Field for revision of the manuscript.

\section{References}

[1] L. Feng, S. Li, Y. Li, H. Li, L. Zhang, J. Zhai, Y. Song, B. Liu, L. Jiang, D. Zhu, Superhydrophobic surfaces: from natural to artificial, Adv. Mater. 14 (2002) 1857-1860.

[2] X. Gao, L. Jiang, Water-repellent legs of water striders, Nature 432 (2004) 36.

[3] Y.-L. Zhang, H. Xia, E. Kim, H.-B. Sun, Recent developments in superhydrophobic surfaces with unique structural and functional properties, Soft Matter 8 (2012) 11217-11231.

[4] R. Blossey, Self-cleaning surfaces — virtual realities, Nat. Mater. 2 (2003) 301.

[5] C.-L. Xu, F. Song, X.-L. Wang, Y.-Z. Wang, Surface modification with hierarchical CuO arrays toward a flexible, durable superhydrophobic and self-cleaning material, Chem. Eng. J. 313 (2017) 1328-1334.

[6] J. Zhang, A. Wang, S. Seeger, Nepenthes pitcher inspired anti-wetting silicone nanofilaments coatings: preparation, unique anti-wetting and self-cleaning behaviors, Adv. Funct. Mater. 24 (2014) 1074-1080.

[7] W.-Y Xie, F. Wang, C. Xu, F. Song, X.-L Wang, Y.-Z Wang, A superhydrophobic and selfcleaning photoluminescent protein film with high weatherability, Chem. Eng. J. 326 (2017) $436-442$. 
[8] Y. Cheng, T. Zhu, S. Li, J. Huang, J. Mao, H. Yang, S. Gao, Z. Chen, Y. Lai, A novel strategy for fabricating robust superhydrophobic fabrics by environmentally-friendly enzyme etching, Chem. Eng. J. 355 (2019) 290-298.

[9] H. Liu, J. Huang, Z. Chen, G. Chen, K.-Q. Zhang, S.S. Al-Deyab, Y. Lai, Robust translucent superhydrophobic PDMS/PMMA film by facile one-step spray for self-cleaning and efficient emulsion separation, Chem. Eng. J. 330 (2017) 26-35.

[10] S. Jung, M.K. Tiwari, N.V. Doan, D. Poulikakos, Mechanism of supercooled droplet freezing on surfaces, Nat. Commun. 3 (2012) 615.

[11] M. Ruan, W. Li, B. Wang, B. Deng, F. Ma, Z. Yu, Preparation and anti-icing behavior of superhydrophobic surfaces on aluminum alloy substrates, Langmuir 29 (2013) 8482-8491.

[12] D. Zang, R. Zhu, W. Zhang, X. Yu, L. Lin, X. Guo, M. Liu, L. Jiang, Corrosion-resistant superhydrophobic coatings on Mg alloy surfaces inspired by lotus seedpod, Adv. Funct. Mater. 27 (2017) 1605446.

[13] Y. Li, L. Zhu, N. Grishkewich, K.C. Tam, J. Yuan, Z. Mao, X. Sui, $\mathrm{CO}_{2}$-responsive cellulose nanofibers aerogels for switchable oil-water separation, ACS Appl. Mater. Interfaces $11(2019)$ 9367-9373.

[14] S. Gao, X. Dong, J. Huang, S. Li, Y. Li, Z. Chen, Y. Lai, Rational construction of highly transparent superhydrophobic coatings based on a non-particle, fluorine-free and water-rich system for versatile oil-water separation, Chem. Eng. J. 333 (2018) 621-629.

[15] L. Zhang, H. Li, X. Lai, X. Su, T. Liang, X. Zeng, Thiolated graphene-based superhydrophobic sponges for oil-water separation, Chem. Eng. J. 316 (2017) 736-743.

[16] R.K. Gupta, G.J. Dunderdale, M.W. England, A. Hozumi, Oil/water separation techniques: a review of recent progresses and future directions, J. Mater. Chem. A 5 (2017) 16025-16058. [17] Z. Chu, S. Seeger, Multifunctional hybrid porous micro-/nanocomposite materials, Adv. Mater. 27 (2015) 7775-7781. 
[18] H. Che, M. Huo, L. Peng, T. Fang, N. Liu, L. Feng, Y. Wei, J. Yuan, $\mathrm{CO}_{2}$-responsive nanofibrous membranes with switchable oil/water wettability, Angew. Chem., Int. Ed. 54 (2015) 8934-8938.

[19] C. Cottin-Bizonne, J.-L. Barrat, L. Bocquet, E. Charlaix, Low-friction flows of liquid at nanopatterned interfaces, Nat. Mater. 2 (2003) 237.

[20] X. Yao, Y. Song, L. Jiang, Applications of bio-inspired special wettable surfaces, Adv. Mater. 23 (2011) 719-734.

[21] J. Lv, Z. Gong, Z. He, J. Yang, Y. Chen, C. Tang, Y. Liu, M. Fan, W.-M. Lau, 3D printing of a mechanically durable superhydrophobic porous membrane for oil-water separation, J. Mater. Chem. A 5 (2017) 12435-12444.

[22] J.T. Han, B.K. Kim, J.S. Woo, J.I. Jang, J.Y. Cho, H.J. Jeong, S.Y. Jeong, S.H. Seo, G.W. Lee, Bioinspired multifunctional superhydrophobic surfaces with carbon-nanotube-based conducting pastes by facile and scalable Printing, ACS Appl. Mater. Interfaces 9 (2017) 77807786.

[23] T. Darmanin, F. Guittard, Recent advances in the potential applications of bioinspired superhydrophobic materials, J. Mater. Chem. A 2 (2014) 16319-16359.

[24] S. Srinivasan, V.K. Praveen, R. Philip, A. Ajayaghosh, Bioinspired superhydrophobic coatings of carbon nanotubes and linear $\pi$ systems based on the "bottom-up" self-assembly approach, Angew. Chem., Int. Ed. 47 (2008) 5750-5754.

[25] P. Zhang, L. Lin, D. Zang, X. Guo, M. Liu, Designing bioinspired anti-biofouling surfaces based on a superwettability strategy, Small 13 (2017) 1503334.

[26] P. Roach, N.J. Shirtcliffe, M.I. Newton, Progess in superhydrophobic surface development, Soft Matter 4 (2008) 224-240.

[27] T. Verho, C. Bower, P. Andrew, S. Franssila, O. Ikkala, R.H.A. Ras, Mechanically durable superhydrophobic surfaces, Adv. Mater. 23 (2011) 673-678. 
[28] N. Wang, Y. Lu, D. Xiong, C.J. Carmalt, I.P. Parkin, Designing durable and flexible superhydrophobic coatings and its application in oil purification, J. Mater. Chem. A 4 (2016) 4107-4116.

[29] Y. Si, Z. Guo, W. Liu, A robust epoxy resins @ stearic acid- $\mathrm{Mg}(\mathrm{OH})_{2}$ micronanosheet superhydrophobic omnipotent protective coating for real-Life applications, ACS Appl. Mater. Interfaces 8 (2016) 16511-16520.

[30] J. Song, D. Zhao, Z. Han, W. Xu, Y. Lu, X. Liu, B. Liu, C.J. Carmalt, X. Deng, I.P. Parkin, Super-robust superhydrophobic concrete, J. Mater. Chem. A 5 (2017) 14542-14550.

[31] C. Su, Y. Xu, F. Gong, F. Wang, C. Li, The abrasion resistance of a superhydrophobic surface comprised of polyurethane elastomer, Soft Matter 6 (2010) 6068-6071.

[32] A. Steele, I. Bayer, E. Loth, Adhesion strength and superhydrophobicity of polyurethane/organoclay nanocomposite coatings, J. Appl. Polym. Sci. 125 (2012) E445-E452. [33] C. Peng, Z. Chen, M.K. Tiwari, All-organic superhydrophobic coatings with mechanochemical robustness and liquid impalement resistance, Nat. Mater. 17 (2018) 355-360. [34] X. Zhu, Z. Zhang, G. Ren, J. Yang, K. Wang, X. Xu, X. Men, X. Zhou, A novel superhydrophobic bulk material, J. Mater. Chem. 22 (2012) 20146-20148.

[35] X. Zhang, Y. Guo, H. Chen, W. Zhu, P. Zhang, A novel damage-tolerant superhydrophobic and superoleophilic material, J. Mater. Chem. A 2 (2014) 9002-9006.

[36] X. Zhang, D. Zhi, L. Sun, Y. Zhao, M.K. Tiwari, C.J. Carmalt, I.P. Parkin, Y. Lu, Superdurable, non-fluorinated superhydrophobic free-standing items, J. Mater. Chem. A 6 (2018) $357-362$

[37] J. Xu, L. Zhang, R. Shi, Y. Zhu, Chemical exfoliation of graphitic carbon nitride for efficient heterogeneous photocatalysis, J. Mater. Chem. A 1 (2013) 14766-14772. 
[38] G. C. Lisensky, D.J. Campbell, K.J. Beckman, C.E. Calderon, P.W. Doolan, R.M. Ottosen, A.B. Ellis, Replication and compression of surface structures with polydimethylsiloxane elastomer, J. Chem. Educ. 76 (1999) 537.

[39] J. Zhang, S. Seeger, Superoleophobic coatings with ultralow sliding angles based on silicone nanofilaments, Angew. Chem., Int. Ed. 50 (2011) 6652-6656.

[40] A.B.D. Cassie, S. Baxter, Wettability of porous surfaces, Trans. Faraday Soc. 40 (1944) $546-551$

[41] X. Zhou, J. Kong, J. Sun, H. Li, C. He, Stable superhydrophobic porous coatings from hybrid ABC triblock copolymers and their anticorrosive performance, ACS Appl. Mater. Interfaces 9 (2017) 30056-30063.

[42] S. Jia, Y. Lu, S. Luo, Y. Qing, Y. Wu, I.P. Parkin, Thermally-induced all-damage-healable superhydrophobic surface with photocatalytic performance from hierarchical $\mathrm{BiOCl}$, Chem. Eng. J. 366 (2019) 439-448.

[43] Y. Wei, Y. Zhu, Y. Jiang, Photocatalytic self-cleaning carbon nitride nanotube intercalated reduced graphene oxide membranes for enhanced water purification, Chem. Eng. J. 356 (2019) 915-925

[44] C.-J. Chen, H.-Y. Lai, C.-C. Lin, J.-S. Wang, R.-K. Chiang, Preparation of monodisperse iron oxide nanoparticles via the synthesis and decomposition of iron fatty acid complexes, Nanoscale Res. Lett. 4 (2009) 1343.

[45] W. Barthlott, C. Neinhuis, Purity of the sacred lotus, or escape from contamination in biological surfaces, Planta 202 (1997) 1-8.

[46] W. Wan, S. Yu, F. Dong, Q. Zhang, Y. Zhou, Efficient $\mathrm{C}_{3} \mathrm{~N}_{4} /$ graphene oxide macroscopic aerogel visible-light photocatalyst, J. Mater. Chem. A 4 (2016) 7823-7829. 
[47] Q. Liu, Y. Guo, Z. Chen, Z. Zhang, X. Fang, Constructing a novel ternary $\mathrm{Fe}(\mathrm{III}) /$ graphene/g- $\mathrm{C}_{3} \mathrm{~N}_{4}$ composite photocatalyst with enhanced visible-light driven photocatalytic activity via interfacial charge transfer effect, Appl. Catal. B 183 (2016) 231-241. [48] F. Dong, L. Wu, Y. Sun, M. Fu, Z. Wu, S.C. Lee, Efficient synthesis of polymeric $\mathrm{g}^{-\mathrm{C}_{3} \mathrm{~N}_{4}}$ layered materials as novel efficient visible light driven photocatalysts, J. Mater. Chem. 21 (2011) $15171-15174$.

[49] X. Miao, X. Shen, J. Wu, Z. Ji, J. Wang, L. Kong, M. Liu, C. Song, Fabrication of an all solid Z-scheme photocatalyst $\mathrm{g}-\mathrm{C}_{3} \mathrm{~N}_{4} / \mathrm{GO} / \mathrm{AgBr}$ with enhanced visible light photocatalytic activity, Appl. Catal. A-Gen 539 (2017) 104-113.

[50] X. Wang, S. Blechert, M. Antonietti, Polymeric graphitic carbon nitride for heterogeneous photocatalysis, ACS Catal. 2 (2012) 1596-1606.

[51] Y. Lu, S. Sathasivam, J. Song, C.R. Crick, C.J. Carmalt, I.P. Parkin, Robust self-cleaning surfaces that function when exposed to either air or oil, Science 347 (2015) 1132-1135.

[52] B. Bhushan, Y.C. Jung, K. Koch, Self-cleaning efficiency of artificial superhydrophobic surfaces, Langmuir 25 (2009) 3240-3248.

[53] S. Liu, X. Zhang, S. Seeger, Solvent-free fabrication of flexible and robust superhydrophobic composite films with hierarchical micro/nanostructures and durable selfcleaning functionality, ACS Appl. Mater. Interfaces 11 (2019) 44691-44699.

[54] T.-S. Wong, S.H. Kang, S.K.Y. Tang, E.J. Smythe, B.D. Hatton, A. Grinthal, J. Aizenberg, Bioinspired self-repairing slippery surfaces with pressure-stable omniphobicity, Nature 477 (2011) 443 . 